\title{
Consistent delay of reward vs consistent nonreward in the alley
}

\author{
J. V. COUCH AND L. R. STANLEY \\ TRINITY UNIVERSITY
}

Albino rats were given training in a runway with either consistent delay or consistent nonreward. The results indicated no performance differences between the two conditions; however, a pretraining effect was evident resulting in superior initial performance for the delay group relative to the nonreward group. This result was interpreted in terms of the Capaldian sequential hypothesis.

Logan (1960) found animals receiving consistent 30 sec delay of reward (CD) show a significant speed increase over days when compared with a consistent nonrewarded (CN) group. Logan adds, however, that the "acquisition"' exhibited may be confounded with drive factors. That is, when pretraining deprivation (five days for Logan) is minimal, increases in performance could be attributed to an unstable hunger cycle.

Stanley \& Kinler (1967), employing twice as many days of deprivation (10 days; three days of goal box placements), found that a consistent $60 \mathrm{sec}$ delayed reward group exhibited no apparent performance increase after the first few days.

The present investigation is an attempt to determine whether the apparent inconsistent findings between the two studies can be attributed to pretraining deprivation differences or delay-confinement differences.

\section{Method}

The Ss were 22 male albino rats, 90-100 days old, obtained from the Holtzman breeding laboratories. The Ss were randomly assigned to two groups of 11 animals each. The apparatus was a gray straight-alley runway, 60 in. long, 4 in. wide, with 9 in. high sides, covered with Plexiglas. An 8 in. floor treadle was located in the start section of the alley and, when depressed by S's weight, started a clock calibrated to $.01 \mathrm{sec}$.

Clock one was stopped (Start time) and clock two started when $S$ interrupted a photobeam 2 in. beyond the treadle. Clock two was stopped (Run time) and clock three started when $S$ broke a second photobeam located 45 in. from the first. A final photobeam (Goal time) was located $12 \mathrm{in}$. beyond the second and 1-1/2 in. in front of a $4 \times 1-1 / 2$ in. wooden partition on the right side of the alley. Behind the partition was located a $1 \times 1 \times 3 / 4$ in. Plexiglas food cup in which $10.045 \mathrm{~g}$ Noyes food pellets were placed on delayed trials. On delayed trials, interruption of the final photobeam by the $S$ activated a timer which automatically opened the food cup lid at the end of a specified delay interval. On nonrewarded trials, the goal cup lid opened immediately upon S's entry to the goal box. A manuallylowered guillotine door served to confine $S$ to the goal section.
On Day 1, animals were placed in individual cages and a 23-h food deprivation schedule was instituted. On Days 3-5, animals were handled in two groups of 11 each for an hour a day with Noyes pellets present. On Day 6, each $S$ was given one goal box placement with the food cup lid open. On Day 7, each $S$ was placed in a neutral waiting box for $30 \mathrm{sec}$, and then given one goal placement, the food cup lid opening automatically upon $S$ being placed in the goal box.

The 30 days of training (one trial/day) began on Day 8. Group $C D$ received $30 \mathrm{sec}$ delay of reward on all trials, while group $\mathrm{CN}$ received consistent $30 \mathrm{sec}$ nonrewarded goal box confinements on all trials. Ss were fed their daily ration $(12 \mathrm{~g})$ approximately $10 \mathrm{~min}$ after the daily trial.

Results

In Fig. 1 is plotted the mean running speed for the two groups in blocks of five trials for the start and goal sections of the alley. In the start section, there was no difference between groups, nor was the Days by Groups interaction significant; however, the Days effect was significant ( $F=10.69, \mathrm{df}=2 / 40, \mathrm{p}<.001$ ) indicating a progressive decline in daily performance. For the goal section, neither Groups nor Days differed, but the interaction was significant ( $F=8.59$, df $=2 / 40, p<.001$ ), indicating a progressive increase for Group $\mathrm{CN}$ and a progressive decrease for Group CD over days. In the run section neither Groups, Days, or the interaction approached significance.

For descriptive purposes, within-group performance in the goal section was analyzed, comparing the first 10 trials with the final 10 trials of training. Group CN's performance increased significantly ( $F=5.90, \mathrm{df}=1 / 10$, $p<.05)$ from the first to last 10 trial block while

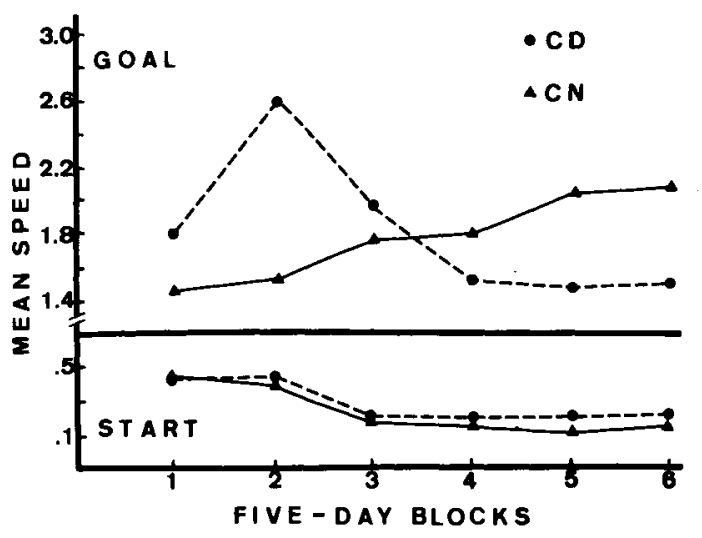

Fig. 1. Mean running speed for each group for the start and goal sections. 
Group CD's performance decreased significantly ( $F$ $=10.90$, df $=1 / 10, p<.01$ ).

Discussion

The results of the present investigation employing a $30 \mathrm{sec}$ delay of reward are consistent with Stanley \& Kinler (1967), but seem only partially consistent with the data reported by Logan (1960). Logan reports that "a slight but distinct 'learning' curve"' was evident for a nonrewarded group when total alley speed was used. This result was replicated in the present investigation, and would appear to indicate, as Logan suggests, progressive adaption of the animals to the experimental situation. Logan reports, however, the greatest gain in performance appearing in the start section with the least gain in the goal section. The present investigation failed to replicate this finding. Both the analyses and Fig. 1 indicate a progressive daily loss for groups $\mathrm{CD}$ and $\mathrm{CN}$ in the start section and a significant increase for $\mathrm{CN}$ and a significant loss for $C D$ in the goal section over days.

The initial increase of speed for the $C D$ group in the goal section, with no similar increase for Group $\mathrm{CN}$, would appear consonant with the sequential hypothesis of Capaldi (1967) and can be interpreted as a pretraining effect (PTE). Consider the stimulus continuum from the sequential hypothesis where the stimulus consequences of the previous trial are arranged from reward $\left(S^{R}\right)$ through delay of reward $\left(S^{D}\right)$ to nonreward $\left(\mathrm{S}^{\mathrm{N}}\right)$. Pretraining goal boxplacements can be considered to result in associative strength at $S^{R}$, which should then generalize along the continuum to $\mathrm{S}^{\mathrm{D}}$ and $\mathrm{S}^{\mathrm{N}}$. Thus, at the beginning of training, it would be expected that Group $C D$, receiving delay trials resulting in $S^{D}$, would have greater generalized habit from $s^{R}$ than would Group $\mathrm{CN}$, with its nonreward trials resulting in the stimulus consequences $S^{N}$, located more remotely on the continuum. Thus $C D$ should be initially superior to $C N$, particularly in the goal section.

With repeated delay trials, stimulus modification should occur and result in modified $S^{D}$ 's more remote from the pretraining $s^{R}$. The progressive decrease in performance in Group CD with days would appear to reflect generalization decrement as the delay trace becomes more remote from the associative strength acquired at $\mathbf{S}^{\mathbf{R}}$.

\section{References}

CAPALDI, E. J. A sequential hypothesis of instrumental learning. In K. W. Spence and J. T. Spence (Eds.), The psychology of learning and motivation: Advances in research and theory. Vol. 1. New York: Academic Press, 1967.

LOGAN, F. A. Incentive. New Haven: Yale University Press, 1960. STANLEY, L. R., \& KINLER, R. M. Recovery following extinction of a locomotor response. Psychon. Sci, 1967, 8, 181-182. 